\title{
Observation of coupled plasmon-polariton modes in Au nanoparticle chain waveguides of different lengths: Estimation of waveguide loss
}

\author{
Stefan A. Maier, ${ }^{\text {a) }}$ Pieter G. Kik, and Harry A. Atwater \\ Thomas J. Watson Laboratory of Applied Physics, California Institute of Technology, Pasadena, \\ California 91125
}

(Received 14 May 2002; accepted for publication 8 July 2002)

\begin{abstract}
Near-field interactions between closely spaced Au nanoparticles were characterized by studying the spectral position of the extinction bands corresponding to longitudinal $(L)$ and transverse $(T)$ plasmon-polariton modes of $\mathrm{Au}$ nanoparticle chains. Far-field spectroscopy and finite-difference time-domain simulations on arrays of $50 \mathrm{~nm}$ diameter Au spheres with an interparticle spacing of 75 $\mathrm{nm}$ both show a splitting $\Delta E$ between the $L$ and $T$ modes that increases with chain length and saturates at a length of seven particles at $\Delta E=65 \mathrm{meV}$. We show that the measured splitting will result in a propagation loss of $3 \mathrm{~dB} / 15 \mathrm{~nm}$ for energy transport. Calculations indicate that this loss can be reduced by at least one order of magnitude by modifying the shape of the constituent particles. () 2002 American Institute of Physics. [DOI: 10.1063/1.1503870]
\end{abstract}

In a recent paper, a method was proposed for guiding electromagnetic energy below the diffraction limit at visible frequencies using ordered arrays of closely spaced noble metal nanoparticles. ${ }^{1}$ Energy transport in these plasmon waveguides relies on near-field coupling between surface plasmon-polariton modes of neighboring particles. In contrast to conventional optical waveguides, the minimum size of the guided modes is not limited by the diffraction limit $\lambda / 2 n$ of light, enabling the fabrication of nanoscale optical devices. This type of guiding due to near-field coupling was recently demonstrated experimentally in macroscopic structures operating in the microwave regime. ${ }^{2}$ At the submicron scale, a theoretical analysis of plasmon waveguides was done using a point-dipole model, allowing for the determination of the dispersion relation $\omega(\mathbf{k})$ and group velocities $v_{g}$ for energy transport. ${ }^{3,4}$ The predictions of the point-dipole model for the collective (in-phase) excitation of the longwavelength mode (wavevector $\mathbf{k}=0$ ) of plasmon waveguides consisting of $80 \mathrm{Au}$ nanoparticles were confirmed using farfield spectroscopy. ${ }^{5}$

Energy transport in plasmon waveguides relies on the excitation of modes with a finite wave vector $(\mathbf{k} \neq 0)$. The functional form $\omega(\mathbf{k})$, the group velocity, and the energy propagation loss all depend on the number of directly interacting nanoparticles. In this letter, we investigate this optical near-field interaction range via a determination of the collective plasmon resonance frequencies for structures with 3, 5, and $7 \mathrm{Au}$ nanoparticles using finite-difference time-domain (FDTD) simulations. The results are compared with far-field extinction measurements on arrays of plasmon waveguides fabricated using electron-beam lithography. Additionally, a simple mathematical formula is deduced relating the far-field extinction data and the expected waveguide loss.

Figure 1 outlines our simulation approach for the determination of the surface plasmon resonance energies $E_{L, T}$ of nanoparticles in plasmon waveguides, where $L$ and $T$ correspond to polarization along (longitudinal mode $L$ ) or perpen-

${ }^{a)}$ Electronic mail: stmaier@caltech.edu dicular (transverse mode $T$ ) to the chain axis. The simulation volume is chosen as a rectangular box of dimensions $1000 \mathrm{~nm} \times 400 \mathrm{~nm} \times 400 \mathrm{~nm}$ and refractive index $n$. The nanoparticle arrays consist of $\mathrm{Au}$ spheres with a diameter $D=50 \mathrm{~nm}$ and a center-to-center spacing $d=75 \mathrm{~nm}$, and the material response of $\mathrm{Au}$ is modeled using a Drude model with a bulk plasmon frequency $\omega_{p}=6.79 \times 10^{15} \mathrm{rad} / \mathrm{s}$ and an electron relaxation time $\tau=4 \mathrm{fs}$. In order to determine the particle resonance frequencies $E_{L, T}$, we initialize the simulation volume with an electrostatic field $\mathbf{E}(t=0)$ polarized either along ( $L$ modes) or perpendicular ( $T$ modes) to the chain axis. The upper inset of Fig. 1 shows $\mathbf{E}(t=0)$ for a particle chain consisting of seven particles for longitudinal polarization. This initial field is chosen to resemble the $\mathbf{k}$ $=0$ waveguide mode, as described in detail elsewhere. ${ }^{6}$ The field is then allowed to evolve for $35 \mathrm{fs}$, and its timedependence $\mathbf{E}(t)$ is analyzed. The simulations produce the correct optical response of a single Au nanoparticle in air, validating the calculation method.

A Fourier transform of the time evolution of $\mathbf{E}(t)$ at the center of each nanoparticle in the arrays allows for the determination of the plasmon resonance energies $E_{L, T}$. Figure 1 shows $\mathbf{E}(t)$ at the center of a nanoparticle located in a seven particle array. The oscillating field decays with a damping constant corresponding to the relaxation time $\tau$ of the Drude model. The Fourier transform of $\mathbf{E}(t)$ for this particular case is shown in the lower inset of Fig. 2 with a clear resonance peak at $2.06 \mathrm{eV}$. Since all particles constituting the arrays are excited in phase, the simulations allow for a determination of the energies of the collective (wave vector $\mathbf{k}=0$ ) plasmon resonance modes.

In order to compare our simulation results with far-field extinction spectra of plasmon waveguides, we fabricated multiple sets of samples consisting of Au nanoparticles with the same diameter $D=50 \mathrm{~nm}$ and center-to-center spacing $d=75 \mathrm{~nm}$ as in the simulation geometry for chain lengths of $3,5,7$, and 80 particles using electron-beam lithography on indium tin oxide coated glass slides. In order to obtain farfield extinction spectra with a high signal-to-noise ratio, 


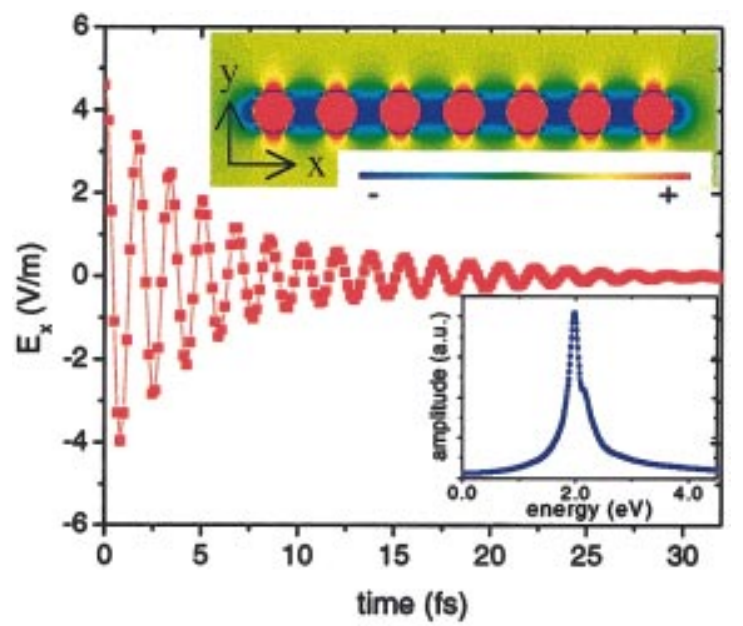

FIG. 1. (Color) Time-dependence $\mathbf{E}(t)$ of the local electric field at the center of a nanoparticle in a seven particle plasmon waveguide as obtained by FDTD simulations. The upper inset shows the distribution of the initial electric field polarized along the chain axis on a linear color scale. The lower inset shows the Fourier transform of $\mathbf{E}(t)$, peaking at the collective plasmon oscillation energy $E_{L}$.

8100 plasmon waveguides of each type were arranged in a $100 \times 100 \mu \mathrm{m}^{2}$ grid with a spacing of $1 \mu \mathrm{m}$, as shown in the scanning electron microscope inset of Fig. 2 for plasmon waveguides consisting of seven nanoparticles. The particles were previously shown to exhibit an almost spherical inplane symmetry. ${ }^{5}$ Crosstalk between adjacent structures is expected to have a negligible effect on the particle interactions since it takes place via far-field scattering of the individual nanoparticles with a distance dependence of $d^{-1}$, whereas near-field interactions of adjacent particles in each waveguide show a $d^{-3}$ dependence and dominate at small distances. ${ }^{8}$ Far-field spectroscopy on these samples can thus be expected to reflect only near-field optical interactions. Transmission measurements on the $100 \times 100 \mu \mathrm{m}^{2}$ grids were performed in an inverted optical microscope with the halogen light source focused to a spot size $<100 \mu \mathrm{m}$, and a polarizer allowed for the selective excitation of the longitudinal or transverse modes. The transmitted light was col-

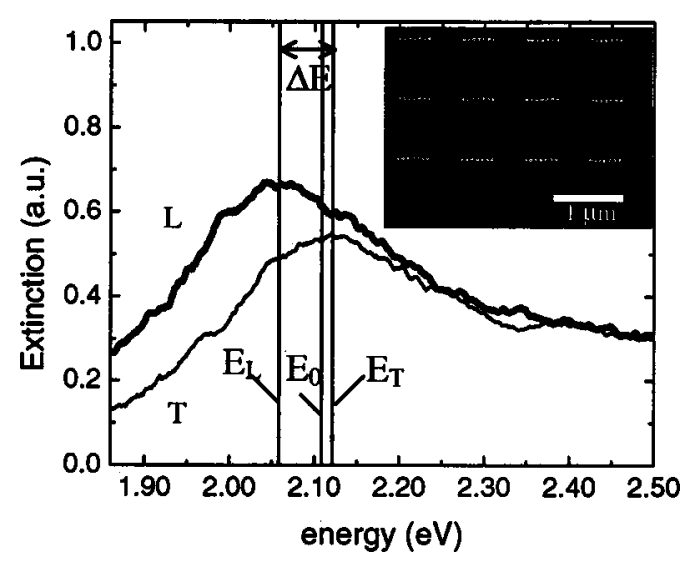

FIG. 2. Far-field extinction spectrum of plasmon waveguides consisting of seven Au nanoparticles for the longitudinal (thick line $L$ ) and the transverse (thin line $T$ ) mode of collective excitation. The peak positions $E_{L}$ and $E_{T}$ are shifted relative to the single-particle plasmon resonance $E_{0}$ due to optical near-field interactions. The inset shows a scanning electron micrograph of

chains with three and five particles suggests that also second-
the plasmon waveguide structures.
Downloaded 03 Apr 2006 to 131.215.225.171. Redistribution subject to AIP license or copyright, see http://apl.aip.org/apl/copyright.jsp

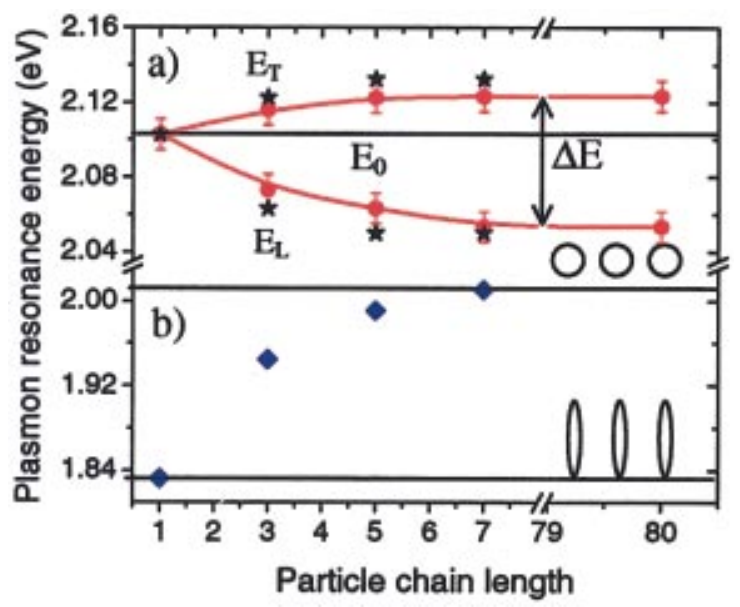

FIG. 3. (Color) (a) Collective plasmon resonance energies for both longitudinal $\left(E_{L}\right)$ and transverse $\left(E_{T}\right)$ excitations for Au nanoparticle arrays of different lengths obtained via far-field spectroscopy (red circles) and FDTD simulations (black stars). (b) Simulation results for the collective plasmon resonance energies for transverse excitation of $\mathrm{Au}$ spheroids with aspect ratios 3:1 (blue diamonds).

lected in a computer-operated microspectrometer and converted to extinction data.

Figure 2 shows a typical far-field extinction spectrum for plasmon waveguides consisting of seven Au nanoparticles taken at normal incidence illumination for both longitudinal (thick line $L$ ) and transverse polarization (thin line $T$ ). The longitudinal extinction peak at $E_{L} \cong 2.06 \mathrm{eV}$ and the transverse peak at $E_{T} \cong 2.12 \mathrm{eV}$ are shifted with respect to the single-particle dipole plasmon resonance at $E_{0} \cong 2.10 \mathrm{eV}$, which was determined on grids consisting of individual nanoparticles with a large interparticle spacing. ${ }^{5}$ The fact that a measurable peak splitting $\Delta E=\left|E_{T}-E_{L}\right|$ occurs between the two extinction peaks indicates that polarizationdependent near-field particle interactions are present. Since our illumination geometry excites all particles constituting the waveguides in phase, the extinction peaks correspond to resonant collective plasmon oscillations of each nanoparticle chain. $^{5}$

Figure 3(a) shows the energies $E_{L, T}$ of the collective modes for plasmon waveguides with $1,3,5,7$, and 80 particles for both $L$ and $T$ polarizations as obtained by far-field experiments (red circles). The peak splitting $\Delta E$ between the longitudinal and the transverse modes increases with particle chain length and saturates at $\Delta E=65 \mathrm{meV}$ for about seven particles. The observed trend in $E_{L, T}$ is in qualitative agreement with Mie-theory calculations for silver nanoparticle chains in air. ${ }^{9,10}$ Figure 3(a) also shows the results of the FDTD simulations described herein (black stars). In order to model the presence of the glass substrate $(n=1.5)$ in the simulations, the medium surrounding the particles was taken to have an effective refractive index $n=1.2$ for the transverse mode. The effective index for the $L$ modes is slightly lower $(n=1.15)$ due to a stronger confinement of the optical near field, which reduces particle-substrate interactions. Experiment and simulations are in good agreement. Note that whereas the dipole model of plasmon waveguides predicts that nearest-neighbor interactions dominate in plasmon waveguides, ${ }^{2}$ the increase in peak splitting between particle chains with three and five particles suggests that also second- 
nearest-neighbor interactions need to be taken into account to correctly describe the waveguide properties.

The peak splitting $\Delta E$ is a direct measurement of the particle interaction strength which in turn determines the bandwidth $B_{L, T}=2\left|E_{0}-E_{L, T}\right|$ available for transport for $L$ and $T$ modes. ${ }^{3,5}$ In our case, $B_{L}=95 \mathrm{meV}(23 \mathrm{THz})$ for the longitudinal mode. The interaction strength also puts an upper limit to the group velocity $v_{g L, T}$ for energy transport, 3,5 and this together with the resistive damping of the oscillation determines the minimum energy loss of propagating waves in plasmon waveguides. This loss was previously estimated to be on the order of $3 \mathrm{~dB} / 500 \mathrm{~nm}$ for small silver particles in air using both a point-dipole model ${ }^{3}$ and calculations based on Mie theory. ${ }^{1}$ The plasmon wave attenuation coefficient $\alpha_{L, T}$ which describes the exponential attenuation of the electric field of the propagating plasmon wave is given by $\alpha_{L, T}$ $=\Gamma / 2 v_{g L, T}$ with $\Gamma$ being the homogeneous linewidth of the plasmon dipole resonance. Using simple algebraic manipulations, it follows that at the point $\mathbf{k}=\pi / 2 d$ of maximum group velocity $\alpha_{L, T} \times d=\Gamma / B_{L, T}$, which can be calculated using quantities obtained from far-field measurements alone. In the case of $50 \mathrm{~nm} \mathrm{Au}$ spheres, $\Gamma=180 \mathrm{meV}$, as obtained directly from the far-field measurements and confirmed using nearfield spectroscopy ${ }^{11}$ corresponding to a decay time $\tau$ of 3.6 fs close to the known decay time of single Au nanospheres. ${ }^{12}$ We thus obtain $\alpha_{L} \approx 2.5 \times 10^{7} \mathrm{~m}^{-1}$ for the fabricated structures with seven nanospheres, corresponding to an energy loss $2 \alpha_{L}$ of about $3 \mathrm{~dB} / 15 \mathrm{~nm}$, showing that the current geometry can not produce long-range energy transfer for the selected materials.

In order to allow for the application of plasmon waveguides in integrated optical circuits, the losses must be decreased. This can be achieved either via a reduction of the homogeneous linewidth $\Gamma$ of the plasmon oscillation or via an increase of the interparticle coupling to increase $\Delta E$ and thus the group velocity $v_{g}$. Both goals can be achieved via a change to materials with a longer electron relaxation time $\tau$ such as $\mathrm{Ag}$ or a change in particle shape. For Au nanoparticles, it has recently been shown that Au nanorods with aspect ratios of 3:1 show a decrease in homogeneous linewidth by a factor 2.25 due to a resonance shift away from the interband transition edge. ${ }^{13}$ To illustrate the effect of changing the particle shape, Figure 3(b) shows FDTD simulation results (blue diamonds) for the transverse collective mode of an array of nanorods approximated with spheroids with their long axis perpendicular to the waveguide chain axis for the same particle volume and center-to-center spacing as in the nanosphere case. The results show a maximum bandwidth
$B_{T}=360 \mathrm{meV}$ for the transverse mode, corresponding to an attenuation coefficient $\alpha=2.7 \times 10^{6} \mathrm{~m}^{-1}$ and an energy loss of $3 \mathrm{~dB} / 130 \mathrm{~nm}$, a factor $\sim 9$ less than in the nanosphere case. Further optimization of waveguide geometry and material is expected to allow for energy propagation over several hundred nanometers.

In summary, we have examined the near-field interaction range of $\mathrm{Au}$ nanoparticles in plasmon waveguides via the determination of the peak splitting $\Delta E$ between the longitudinal and transverse plasmon-polariton collective mode excitations for waveguides of different lengths. We have shown that far-field measurements allow for a quick estimate of the minimum waveguide loss via a determination of the peak splitting $\Delta E$ and the homogeneous linewidth $\Gamma$ of the plasmon oscillations, requiring no near-field measurements. With appropriate geometries and materials, plasmon waveguides should allow for efficient guiding of electromagnetic energy over distances of several hundred nanometers, opening the way for the fabrication of highly integrated optical devices operating below the diffraction limit of light.

The authors would like to thank Richard Muller and Paul Maker of the Jet Propulsion Laboratory in Pasadena for expert technical assistance. This work was sponsored by the NSF (Grant No. ECS0103543) and the Center for Science, Engineering of Materials at Caltech, and the Air Force Office of Scientific Research.

${ }^{1}$ M. Quinten, A. Leitner, J. R. Krenn, and F. R. Aussenegg, Opt. Lett. 23, 1331 (1998).

${ }^{2}$ S. A. Maier, M. L. Brongersma, and H. A. Atwater, Appl. Phys. Lett. 78, 16 (2001).

${ }^{3}$ M. L. Brongersma, J. W. Hartman, and H. A. Atwater, Phys. Rev. B 62, R16356 (2000).

${ }^{4}$ S. A. Maier, M. L. Brongersma, P. G. Kik, S. Meltzer, A. A. G. Requicha, and Harry A. Atwater, Adv. Mater. 13, 1501 (2001).

${ }^{5}$ S. A. Maier, M. L. Brongersma, P. G. Kik, and H. A. Atwater, Phys. Rev. B 65, 193408 (2002).

${ }^{6}$ S. A. Maier, P. G. Kik, and H. A. Atwater, Mater. Res. Soc. Symp. Proc. 722, L6.2 (2002).

${ }^{7}$ U. Kreibig and M. Vollmer, Optical Properties of Metal Clusters (Springer, Berlin, 1994).

${ }^{8}$ B. Lamprecht, G. Schider, R. T. Lechner, H. Ditlbacher, J. R. Krenn, A. Leitner, and F. R. Aussenegg, Phys. Rev. Lett. 84, 4721 (2000).

${ }^{9}$ J. M. Gerardy and M. Ausloos, Phys. Rev. B 25, 4204 (1982).

${ }^{10}$ M. Quinten and U. Kreibig, Appl. Opt. 32, 6173 (1993).

${ }^{11}$ S. A. Maier, P. G. Kik, H. A. Atwater, S. Meltzer, A. A. G. Requicha, and B. E. Koel, Proc. SPIE 4810 (2002) (to be published).

${ }^{12}$ T. Klar, M. Perner, S. Grosse, G. von Plessen, W. Spirkl, and J. Feldmann, Phys. Rev. Lett. 80, 4249 (1998).

${ }^{13}$ C. Sönnichsen, T. Franzl, T. Wilk, G. von Plessen, and J. Feldmann, Phys. Rev. Lett. 88, 077402 (2002). 